\title{
Comparing Various Type of Natural Fibers as Filler in TPU: Mechanical Properties, Morphological and Oil Absorption Behavior
}

\author{
Nor Azwin Ahad ${ }^{1, *}$, Nur Fatin Hidayu Mohd $\operatorname{Radzi}^{1}$, Nor Suhaida Sadli ${ }^{1}$ and Tan Jia Seng ${ }^{1}$ \\ ${ }^{1}$ School of Materials Engineering, Universiti Malaysia Perlis, Kompleks Pusat Pengajian Jejawi 2, \\ 02600, Jejawi,Perlis, Malaysia.
}

\begin{abstract}
The idea of using natural fibers as filler in various polymers has been extensively studied. Various types of natural fibers and polymers have been identified and it can be varied according to the particular application and the two main composite materials will have advantages and disadvantages of each. However, natural fibers are usually selected as filler because it is readily available and environmentally friendly, inexpensive, non-toxic, biodegradable and still have good characteristics for a variety of uses. In this study, four types of natural fiber have been used which; coconut shell, coconut fiber, corn cob, and pineapple skin, as fillers in thermoplastic polyurethane (TPU). The mixing process conducted through melt mixing techniques. The percentage of TPU and natural fibers are 100/0, 95/5, 90/10 and 85/15. Different type of fiber will affect the mechanical properties of the composites and have been studied through tensile testing. It showed that the result for pineapple fiber at 5\% was the highest and can also be related to the characterizations of this composite that have been studied via the SEM morphology. Swelling testing is also having been done to prove the absorbency ability by natural fiber composites in cooking oil and engine oil. Then it concluded that the pineapple fiber absorbed large amount of both oil compared to others.
\end{abstract}

\section{Introduction}

Natural fibers play an important role in developing high performance biodegradable composites which are the key material in solving nowadays environmental and $3 \mathrm{R}$ (Recycle-Reuse-Reduce) issues, because due to its low cost as well as easy to degrade [1]. The natural fibers are the one of popular resource that uses as reinforcement or filler in polymer matrix $[2,3]$. Coconut husk and shell powder, pineapple fiber and corn cob are some of the sources of natural fibers that have potential to use as filler, which have been studied here and blended with TPU. There are several recent studies that combined the natural fiber and TPU, found it to produce some of the unique properties, such as TPU/kenaf by El-Shekeil et al. [4] and Janus \& Patrycja [5]. They found that, kenaf fiber

* Corresponding author: norazwin@unimap.edu.my 
were compatible with TPU from the view of mechanical properties. El-Shekeil et al. [4] studied TPU/cocoa pod husk and proved that, the tensile properties improved when the filler loading increased. Many researchers have been studied natural fiber in polymer composites and it's mechanical, morphological properties in order to investigate the compatibility of fiber/polymer composites. Due to that, we investigated the effect of four types of natural fiber as filler in TPU, with different content of filler $(0 \%, 5 \%, 10 \%$ and $15 \%)$ on tensile strength and the morphology. Also, we determined the swelling percentage of the composite for the optimum content of filler in cooking oil and engine oil, to study the level of oil absorption by the composite of the four types of filler.

\section{Experimental}

The preparation of TPU composite with natural fibers ass filler in this study were done in polymer laboratory and material characteristics laboratory in School of Materials Enginering in Universiti Malaysia Perlis within 10 months schedule.

\subsection{Materials}

Polymer-based TPU in the pallet form was supplied from Duplas Marketing Sdn. Bhd. All natural fibers (pineapple, coconut husk, coconut shell and corncob) were collected from market, stall and village. They were cut into small size, washed and dried under direct sunlight for days to evaporate all the moisture. The dried fibers was grinded using grinding machine to produce fibers in the small size and were sieved using sieve shaker to get fine size between the range of $50-125 \mu \mathrm{m}$.

\subsection{Preparation of TPU/ natural fiber composites}

The composite were prepared by melt blending technique using laboratory internal mixer (RheoHaake 600p) at the weight percent of 100/0, 95/5, 90/10 and 85/15 for TPU and natural fiber. Blending was carried out at temperature $180^{\circ} \mathrm{C}$, with a mixing speed of 60 $\mathrm{rpm}$ for $10 \mathrm{~min}$ [6]. Then it was pressed at $185^{\circ} \mathrm{C}$ under $45 \mathrm{MPa}$ of pressure for about $2 \mathrm{~min}$ using hot press (Carver Laboratory Press) into thin sheet. The composite sheet then cut into dumbbell shape for tensile testing. The rest of the samples were prepared for swelling test.

\subsection{Characterization of TPU/natural fibers composites}

Tensile testing is to measure the tensile properties and was done using an Instron Testing Machine. The thin sheets of the sample were about $3 \mathrm{~mm}$ according to ASTM 638. Oil absorption test have been done to prove the ability of the composites to absorb cooking oil and engine oil.The samples with measurements of $20 \mathrm{~mm} \times 20 \mathrm{~mm} \times 1 \mathrm{~mm}$ were used as indicted by ASTM D570-98. The test have been observed and determined for 4 weeks until equilibrium at room temperature. The samples were weighted for everyday and calculated using the equation of;

$$
\text { Oil absorption }(\%)=\frac{\text { intial weight }- \text { final weight }}{\text { final weight }} \times 100 \%
$$

Scanning electron microscopy (SEM) was used to evaluate fiber's dispersion in the TPU matrix and to illustrate the fiber morphology of all the type of the fibers used. This testing 
which using SEM HITACHI TM 300, was also to observe and investigate the morphology of the tensile fracture surface of the composites after tensile testing.

\section{Results}

The results will be presented here are on the tensile strength, oil absorption percentage and morphology of the composites that have been studied.

\subsection{Tensile strength}

The tensile strength of the TPU composites with various type of natural fiber can be inferred in Fig. 1. This figure illustrated the evolution of the tensile strength and a marked highest was observed at $5 \%$ of pineapple fiber, $10 \%$ of coconut husk, $15 \%$ of coconut shell and $10 \%$ of corncob, when we not compared it to the neat TPU sample. Also, we can consider these are the optimum filler loading for each type of natural fiber used in TPU. Due to various type of fiber, it can be seen that the tensile strength is affected by filler type, filler size, filler shape and their surface area [7]. It would also be controlled by the nature of the filler and matrix [8]. By comparing four natural fibers in this study, we may proved that pineapple fiber showed the highest tensile strength among others, although with low filler loading (5\%) compared to other. Even, Kengkhetkit et al. [9] in their study resulted that the tensile strength show an increasing when filler at the range of $10 \%$-to $30 \%$. This may be due to the TPU, which have better interaction with hydroxyl groups on cellulosic fiber [10]. Beside, at higher loading of coconut shell (15\%), the composite showed the highest tensile strength. This is due to the shape of coconut shell itself. The more loading are needed to make the distribution of filler become well enough and good interaction with the TPU matrix. Georgopoulos et al. [11] stated that, plant fibers are not easily dispersed in nonpolar polymer due to their extensive intermolecular hydrogen-bonding. They also found in previous research, the decrease in tensile strength occurs upon loading the polymer with natural fiber. This fact also was supported by Myrath et al. [7]. They found that, the addition of natural fibers into the polymer, decreases tensile strength and this is the common observation with almost all filled polymer system. The decreasing in tensile properties of composites causes by the poor fiber dispersion. Consequently, the higher the cellulose content for each type of natural fiber (Table 1), the higher the tensile strength. Youssef et al. [1] and Georgopoulos et al. [11] proved that, high tensile strength and more obvious at the higher fiber loading of $10 \%$ corncob fiber, the value decreased. Referred to them, the decreasing may be due to decreasing of the internal bond. Differ to the coconut husk as filler (10\%), resulting in an increasing of tensile strength and this is justified by Emmanuel et al. [3] which, with the enhancement of the interfacial adhesion and the ability to transfer stresses across the interface. Other possible reason is, some researchers proved that, most natural fibers start to lose strength at $160^{\circ} \mathrm{C}$, which expected to cause poor mechanical properties in the final products [11]. This may be seen from the result of tensile strength. 


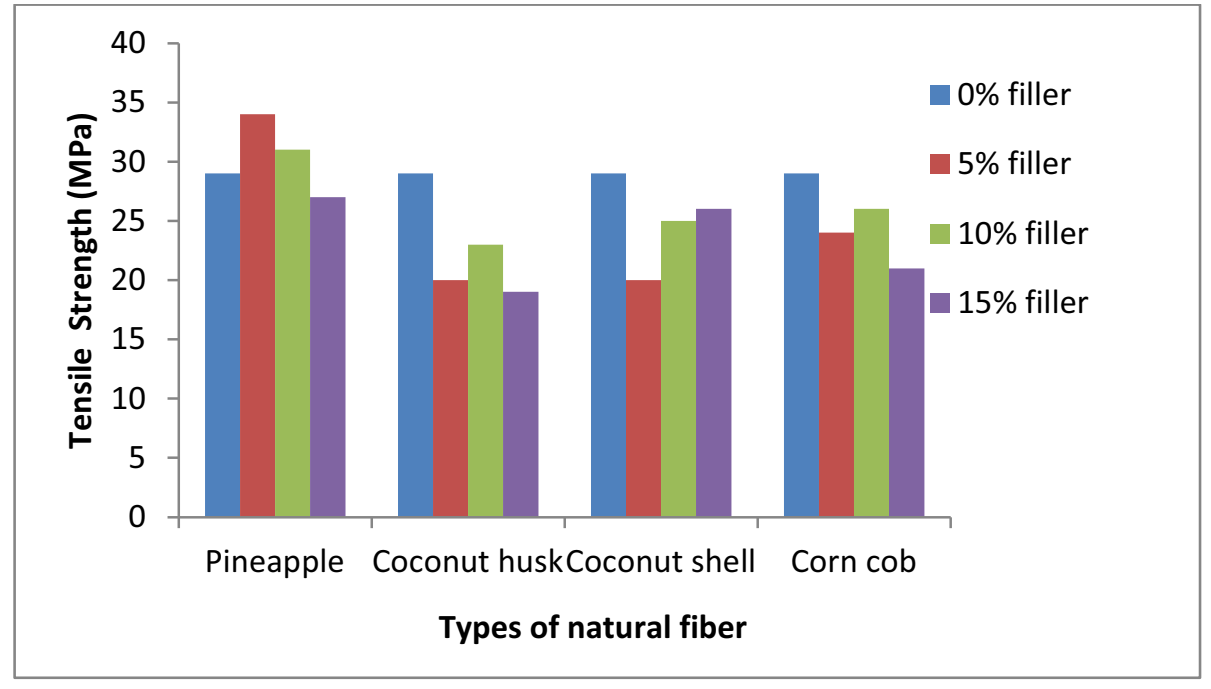

Fig. 1. Tensile strength for natural fiber/TPU composites at $0 \%-15 \%$ of filler content.

\subsection{Oil absorption behavior}

The pattern of oil absorption after 4 weeks by the TPU composite with different types of natural fibers can be referenced in Fig. 2. All fibers showed higher oil absorption capacity for cooking oil compared to engine oil. Cooking oil has low viscosity so that the oil molecules can move easily to the pores of the fiber assembly and hence higher absorption capacity was observed. We may see that, coconut shell and corncob fiber have the lowest oil absorption capacity. Due to Marko et al. [12], several types of fibers have some drawback like oil absorption capacity due to its oleophobicity properties. Other reason is due to their shape, surface area and their nature. By looking to the shape and nature of filler, we can say that pineapple fiber and coconut husk contain long and fine fibrous structure which can easy access of the oil [9]. Coconut shell as we know, are in powder form with irregular shape and corncob contain only short fibers. Strong fiber and matrix interfacial adhesion also can help to diminish the oil penetration and avoiding the worsening of the mechanical performance of the composite, which we may relate to the tensile strength in previous discussion. Long fiber with hollow tubes structures may serves a valuable for oil absorption. Jayamol et al. [13] stated that, if the surface area is large, then there will be more area for absorption of oil molecules. Surface cavities are filled with oil molecules that have been absorbed. Furthermore, the percentage of oil absorption and swelling which obtained with different type of oil are determined by the ability of the oil to interact with the cellulosic fiber. The efficiency of the absorption process also depends on the packing density of the filler, but this is not yet to be determined in this study. From Emmanuel et al. [3] research, fiber assemblies with lower packing density are more porous and have a large hydraulic diameter of inter-fiber pores. The density of the oil used also influences the oil absorption capacity. 


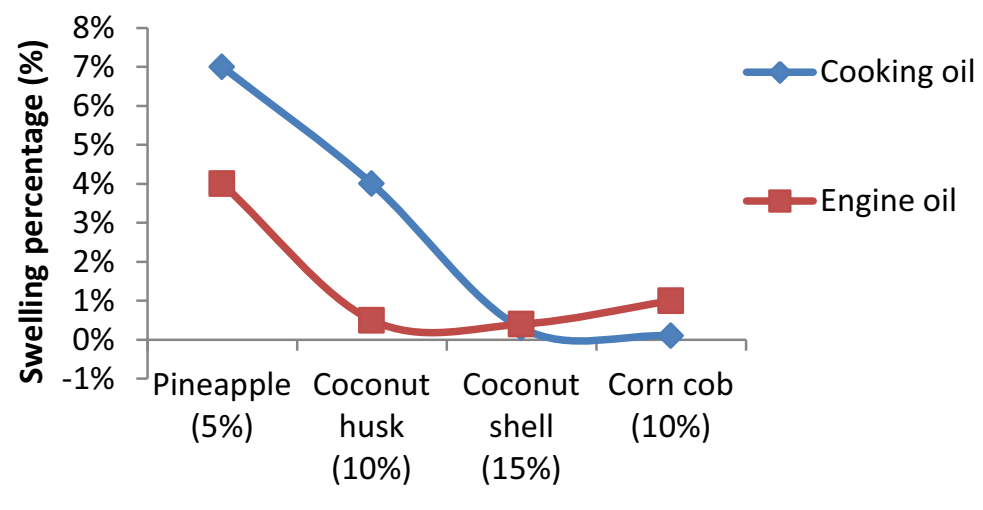

Types of natural fiber at its optimum filler content

Fig. 2. Swelling percentage for natural fiber/TPU composites at optimum filler content, in cooking oil and engine oil after 4 weeks.

\subsection{Morphology}

Every natural fiber has its own surface morphology which decides the interfacial matrixfiber adhesion and these will affected the mechanical/physical properties [14]. Cellulose content affects the mechanical properties of vegetable and fruits fiber [11]. Short fiber leads to higher composites strength, most probably due to better dispersion which gives anisotropic products. While particulate filler is dispersed into polymer melt and quite difficult to achieve a strong bond between particles and matrix. It is just because the poor wettability of the fine filler particles by the polymer melts. Since the particulate filler are very small, a high interfacial area with filler and the polar matrix. This area increases the worsening bonding between them and decreases the tensile strength. Irregular shape filler such as corncob [15] can caused to the decreases of strength of this composites. The cellulose content for all four types of natural fibers is presented in Table 1. Cellulose provides strength and stability to the cell walls and the fibers as a whole. The cellulose oriented in a fiber or bundle of fibers influences its properties [16]. Fig. 3 (a) showed the smooth surface of matrix and this described the compatibility between TPU matrix and pineapple fibers. Due to this good interfacial adhesion, it proved to the highest tensile strength [10]. Fig. 3 (b,c,d) showed the rough surface with the presences of fibers revealed less homogeneity [1]. The fibers also appear nearly to be free of the matrix material adhering to them. Also, could be seen, some cavities between fibers and the TPU component. The morphology of natural fibers reveals shapes in the form of long fiber, short fiber and also irregular particles.

Table 1. Cellulose content of natural fibers [17, 18].

\begin{tabular}{|c|c|}
\hline Natural fiber: & Cellulose content (\%): \\
\hline Pineapple & $73-81 \%$ \\
\hline Coconut husk & $32-43 \%$ \\
\hline Coconut shell & $34 \%$ \\
\hline Corncob & $31 \%$ \\
\hline
\end{tabular}



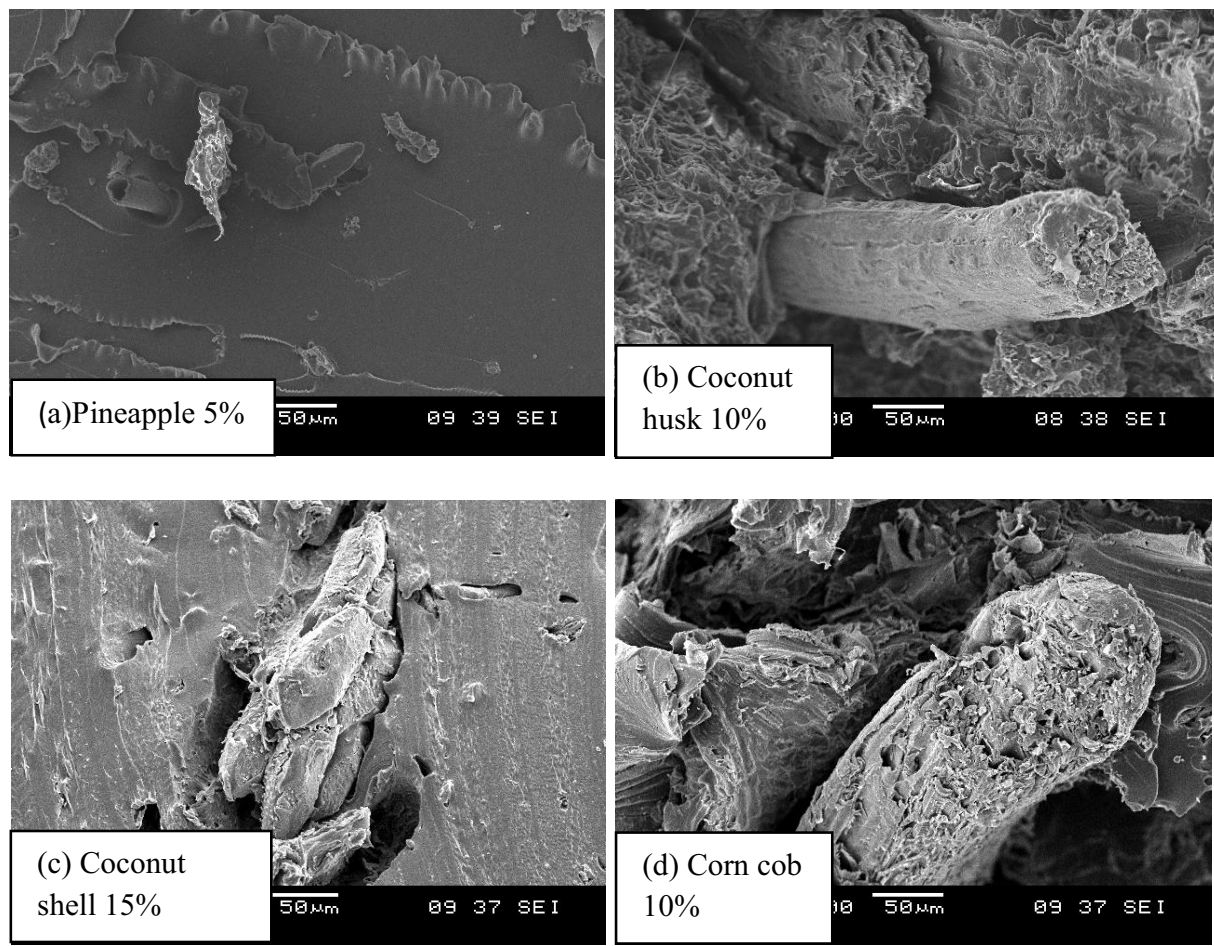

Fig. 3. Morphology for natural fiber/TPU composites at their optimum filler content; (a) Pineapple fiber at 5\% loading (b) Coconut husk at 10\% loading (c) Coconut shell powder at $15 \%$ loading and (d) corncob fiber at $10 \%$ loading.

At higher filler loadings, agglomerations of fibers caused poorer transfer of load and stresses by fiber agglomerates to matrix, which decreased the mechanical properties of composites. The composites which failed are mostly by fiber pull out from the matrix and indicate a poor interfacial bonding between the matrix and fibers [14]. Voids on the surface are also quite visible. Voids arise due to the entrapped air pocket in the matrix during composites processing [14].

\section{Conclusions}

Fruit and plant fiber are often seen as waste produced in the agricultural sector and can be transformed into profitable, useable products. The main characteristics of the most natural fibers however, are described in terms of their mechanical properties such as tensile strength. Among the four types of natural fibers been studied, pineapple at 5\% filler content showed the highest strength and it proved by the composite's morphology. Good interaction between filler and matrix can be observed to the fiber break up and the surface roughness of the matrix. Different type of natural fiber represented different morphology and also displayed different tendency to absorb oil. Highest swelling percentage represented a highest level of TPU composite to absorb the both types oil with the incorporation of pineapple fibers. 


\section{References}

1. A.M. Youssef, A. El Gendy, S. Kamel, Mater. Chem. Phys., 152, 26 (2015)

2. G.J. Nilza, G.J. Virgo, V.E. Buchanan, Mater. Char., 59, 1273 (2008)

3. M.F. Emmanuel, V.M. Correlo, J.F. Mani, R.L. Reis, Com. Sci. Tech., 78, 56 (2013)

4. Y.A. El Shekeil, S.M. Sapuan, M.W. Algrafi, Mater. Des., 64, 330 (2014)

5. D. Janusz, K. Patrycja, Indstl. Crops and Prods., 74, 566 (2015)

6. A.A. Nor, S.H. Ahmad, N.M. Zain, Appl. Mech. Mater., 291, 2654 (2013)

7. K. Myrath, H. Onggo A. Syampurwadi, J. Bio. Sci., 7 (2), 393 (2007)

8. H.M. da Costa, R.C.R Nunes, L.L.Y. Visconte, C.R.G. Furtado, Raw Mater. App., 54, $242(2001)$

9. N. Kengkhetkit, T. Amornsakchai, Mater. Des., 55, 292 (2014)

10. A. Elkhaoulani, F.Z. Arrakhiz, K. Benmoussa, R. Bouhfid, A. Qaiss, Mater. Des., 49, 203 (2013)

11. Georgopolous, Tarantili, Augerinos, Andrepolous, Polym. Degr. Stab., 90, 303 (2005)

12. L. Marko, M. Remskar, V. Ducman, F. Svegl, J. of Environ. Manage., 114, 158 (2013)

13. G. Jayamol, S.S Bhagawan, S. Thomas, Comp. Sci. Tech., 58, 1471 (1998)

14. R.P. Avinash, R.N. Jagtap, J. Mater. Environ. Sci., 6, 902 (2015)

15. G. Junjie, A.H. Milford, Indstl. Crops Prods., 19, 225 (2004)

16. N. Sena, A.R. Marco, A.M. Araujo, V.D. Souza, L.H.C. Mattaso, J.M. Marconcini, Indstl. Crops Prods., 43, 529 (2013)

17. D. Rudi, K. Myrtha, A.S. Subyakto, D. Hermawan, A. Hadiyane, A. J.of Plant Sci., 15, $42(2016)$

18. A.K. Bledzki, A.A. Mamun, J. Volk, Comp. Sci. Tech., 70840 (2010) 